\title{
Reprogramming of host glutamine metabolism during Chlamydia trachomatis infection and its key role in peptidoglycan synthesis
}

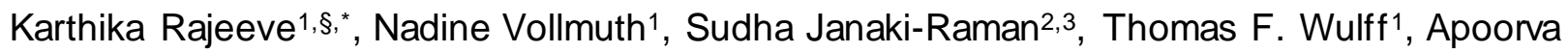
Baluapuri ${ }^{3}$, Francesca R. Dejure ${ }^{3}$, Claudia Huber ${ }^{4}$, Julian Fink ${ }^{6}$, Maximilian Schmalhofer ${ }^{4}$, Werner Schmitz2,3, Rajeeve Sivadasan ${ }^{5}$, Elmar Wolf ${ }^{3}$, Wolfgang Eisenreich ${ }^{4}$, Almut Schulze,\#, Jürgen Seibel ${ }^{6}$, Thomas Rudel ${ }^{1,7^{*}}$

1 Department of Microbiology, Biocenter, University of Würzburg, Am Hubland, D-97074 Würzburg, Germany.

2 Department of Biochemistry and Molecular Biology, Biocenter, University of Würzburg, Am Hubland, D-97074 Würzburg, Germany.

${ }^{3}$ Cancer Systems Biology Group, Department of Biochemistry and Molecular Biology, Biocenter, University of Würzburg, Am Hubland, 97074 Würzburg, Germany.

${ }^{4}$ Lehrstuhl für Biochemie, Technische Universität München, Lichtenbergstr. 4, D-85747 Garching, Germany.

${ }^{5}$ German Cancer Research Center (DKFZ), Im Neuenheimer Feld 280, D-69120, Heidelberg, Germany.

${ }^{6}$ Institute of Organic Chemistry, University of Würzburg, Am Hubland, D-97074 Würzburg, Germany.

7 Helmholtz Institute for RNA-based Infection Research (HIRI), D-97074 Würzburg, Germany.

$\S$ Department of Biomedicine, The Skou Building, Aarhus University, Høegh-Guldbergs Gade 10, DK-8000, Aarhus C, Denmark.

\# BioMed X Innovation Center, Im Neuenheimer Feld 583, D-69120 Heidelberg, Germany.

*For correspondence: karaj@biomed.au.dk, thomas.rudel@biozentrum.uni-wuerzburg.de. 
41 Abstract

43 Obligate intracellular bacteria like Chlamydia trachomatis undergo a complex

44 developmental cycle between infectious non-replicative (EBs) and non-infectious

45 replicative (RBs) forms. EBs shortly after entering a host cell transform to RBs, a crucial

46 process in infection, initiating chlamydial replication. As Chlamydia fail to replicate

47 outside the host cell it is currently unknown how the replicative part of the developmental

48 cycle is initiated. Here we show in a cell-free approach in axenic media that uptake of

49 glutamine by the bacteria is crucial for peptidoglycan synthesis which has a role in

50 Chlamydia replication. The increased requirement for glutamine in infected cells is

51 satisfied by reprogramming the glutamine metabolism in a c-Myc-dependent manner.

52 Glutamine is effectively taken up by the glutamine transporter SLC1A5 and metabolized

53 via glutaminase. Interference with this metabolic reprogramming limited growth of

54 Chlamydia. Intriguingly, Chlamydia failed to produce progeny in SLC1A5 knockout

55 organoids and mice. Thus, we report on the central role of glutamine for the development

56 of an obligate intracellular pathogenic bacterium and the reprogramming of host

57 glutamine metabolism, which may provide a basis for innovative anti-infective strategies. 
60 Chlamydia trachomatis is an obligate intracellular bacterium and the most frequent cause of

61 bacterial sexually transmitted disease ${ }^{1}$. In a biphasic developmental cycle, EBs enter the host

62 cell and convert into RBs, which replicate in a membrane-enclosed structure called "the

63 inclusion". Later, RBs convert back into EBs, which can readily infect neighbouring cells. For a

64 long time, it was thought that Chlamydia do not form peptidoglycan until peptidoglycan ring-like

65 structures were recently discovered exclusively in the septa of dividing RBs ${ }^{2,3}$.

66 Due to its obligate intracellular lifestyle and an evolutionary reduced genome ${ }^{4}$, Chlamydia

67 requires multiple metabolites from the host cell. Nevertheless, this pathogen lacks the enzyme

68 hexokinase but encodes the enzymes required to utilize D-glucose-6-phosphate (G6P) and to

69 convert it into pyruvate via glycolysis ${ }^{5}$. Thus, the bacteria depend on G6P from the host cell. In

70 EBs, ATP is generated from G6P either via glycolysis or oxidative phosphorylation, whereas in

71 RBs ATP is provided by the host cell, while G6P is nearly exclusively used for cell wall

72 biosynthesis ${ }^{6,7}$. The TCA cycle of Chlamydia is incomplete due to the absence of three key

73 enzymes, citrate synthase, aconitase and isocitrate dehydrogenase. This incomplete metabolic

74 pathway must be complemented by the constant metabolic supply from the host ${ }^{4}$. We have

75 previously shown that $C$. trachomatis takes up host-derived malate to feed the partial TCA cycle

$76{ }^{6}$. In addition, amino acids have to be acquired from the host cells due to the limited capacity of

77 Chlamydia to synthesize amino acids ${ }^{6}$. Despite the advancements in the knowledge about 78 chlamydial metabolism, the processes initiating the transition of EBs to RBs after entry into cells 79 are still unknown.

\section{$81 \quad$ Results}

\section{Glutamine metabolism in Chlamydia: A pathway to peptidoglycan biosynthesis}

83 In our attempts to further define the metabolic interaction of Chlamydia and host cells, we 84 performed metabolite uptake assays in axenic culture. Since glutamine (Gln), glutamate (Glu) 85 and $\alpha$-ketoglutarate $(\alpha-K G)$ were previously predicted to feed the partial chlamydial TCA cycle $864,6,8$, we investigated whether these metabolites are directly taken up by Chlamydia (Fig. 1a,b). 
87 Whereas the amount of Gln decreased rapidly in the supernatant, neither Glu nor $\alpha-\mathrm{KG}$ were

88 significantly consumed (Fig. 1c). Using isotopologue profiling with Gln containing only ${ }^{13} \mathrm{C}$ 89 instead of ${ }^{12} \mathrm{C}$ atoms ([U-13 $\left.\mathrm{C}_{5}\right] \mathrm{Gln}$ ) added to the axenic culture, GC-MS analyses of chlamydial 90 extracts revealed that the ${ }^{13} \mathrm{C}$ label is efficiently transferred to Glu, Asp and intermediates of the 91 partial TCA cycle, with the ${ }^{13} \mathrm{C}$-labelled fractions ranging from $80 \%$ in Gin to less than $3 \%$ in 92 pyruvate (Fig. 1d). Substantial fractions of ${ }^{13} \mathrm{C}$-labelling in Glu (60\%), Asp (50\%), succinate 93 (20\%), fumarate (35\%), and malate (8\%) (Fig. 1d) indicated that exogenous Gln is metabolized 94 via glutaminolysis and the TCA cycle to generate Glu, $\alpha-K G$, succinate, fumarate, malate, 95 oxaloacetate and aspartate. Consistently, the amount of chlamydial NADH probably formed by 96 the conversion of $\alpha-K G$ into succinate and malate into oxaloacetate within the truncated 97 chlamydial TCA cycle (Fig. 1a) is significantly increased when exogenous Gln is added to the axenic medium (Extended data Fig. 1a).

99 Interestingly, significant ${ }^{13} \mathrm{C}$-labelling was also detected in Ala (17\%) and the peptidoglycan 100 precursor, diaminopimelate (DAP) (41\%) (Fig. 1d). Whereas Ala contained a high fraction of the $101 \mathrm{M}+3$ isotopologue (i.e. a molecule with three ${ }^{13} \mathrm{C}$-labelled carbon atoms), DAP contained $\mathrm{M}+4$ 102 as main compound, accompanied by $M+2, M+3$, and $M+7$ isotopologues (Fig. $1 \mathrm{~d}$ ). The detected 103 isotopologue composition of Ala can be explained by its formation from pyruvate derived from 104 oxaloacetate via PEP catalysed by PEP carboxykinase and pyruvate kinase; an alternative 105 could be the decarboxylation of Asp, although no gene with the required enzymatic activity is 106 annotated in the genome of Chlamydia (scheme in Extended data Fig. 1b). The labelling pattern 107 in DAP points at its formation from Asp and pyruvate contributing four and three labelled carbon 108 atoms, respectively, and resulting in the detected $M+3, M+4$ and $M+7$ isotopologues species 109 (Fig. 1d, Extended data Fig. 1b). Minor isotopologues especially M+2 in aspartate and DAP can 110 be explained by metabolic cycling via gluconeogensis and the PPP, which both are active in 111 Chlamydia. To further support the unexpected finding of chlamydial DAP synthesis in axenic 112 media, we performed an approximate quantification of DAP (for details see Methods) and could 113 estimate a value of about 0.04 mg DAP formation per gram cells of Chlamydia in the presence 114 of Gln in axenic medium (Extended data Fig. 1c). We also supplied axenic cultures with Gln that 
115 was either labelled in the amino group or the amido group. The ${ }^{15} \mathrm{~N}$-label was only transferred

116 from the amino group of Gln (Extended data Fig. 1d). Together, these results indicated that Gin

117 serves as a major carbon source for the fuelling of the partial TCA cycle of Chlamydia and as a

118 source of amino groups for the generation of amino acids from alpha-keto acids via

119 transamination, e.g. Glu, Asp and Ala, required for peptidoglycan biosynthesis.

120 Synthesis of DAP in axenic medium was particularly intriguing since peptidoglycan synthesis is 121 restricted to the replicative phase of Chlamydia and this has never been observed for Chlamydia 122 outside their host cell ${ }^{2}$. We therefore analysed EBs from axenic cultures in the presence or 123 absence of Gln by electron microscopy (EM). Surprisingly, incubation of EBs with Gln, but not 124 Glu led to a gradual time-dependent morphological transition to an intermediate between EBs 125 and RBs, so-called intermediate bodies (IBs) (Fig. 1e; Extended data Fig. 1e). The infectivity of 126 EBs incubated with GIn dropped to 20\% (Extended data Fig. 1f), supporting the conclusion that 127 Gln induces the developmental transition of EBs to a non-infectious form.

128 We then synthesized a clickable form of the dipeptide DA-DA to detect peptidoglycan in these 129 bacteria $^{2}$ (see Methods). Chlamydial particles incubated in the presence of GIn in this cell-free 130 approach produced detectable peptidoglycan which could be quantified by FACS (Fig. 1f, g, 131 Extended data Fig. 1g). Since peptidoglycan synthesis in Chlamydia has been shown to be 132 associated with replication in host cells, we also investigated if Gln could initiate transcription in 133 Chlamydia in axenic culture. RNA-seq analysis revealed activation of several genes upon 134 exposure to glutamine, including those previously described as early genes ${ }^{9}$ (Fig. $1 \mathrm{~h}$, Extended 135 data Fig. 1h). This finding was also confirmed by quantitative PCR (qPCR) of several genes 136 already reported to be expressed at early time points of EB to RB conversion ${ }^{10}$ (Extended data 137 Fig. 1i). Interestingly, in the presence of Gln, a significant increase in the copy number of 138 chlamydial DNA was detected in axenic media (Fig. 1i), indicating that Gln access initiates 139 limited replication in Chlamydia. 
142 Since our data suggested uptake of Gln as a central node for the interaction between host and

143 bacterial metabolism, we investigated how Chlamydia infection influences the metabolism of

144 primary human umbilical vein endothelial cells (HUVECs). Chlamydia infection induced an 145 increased depletion of most amino acids from the culture medium, indicative of their enhanced uptake by infected cells (Fig. 2a). Gln was one of the most depleted amino acids in the medium

147 (Fig. 2a) and Gln but not Glu uptake was significantly increased in infected cells (Fig. 2b,c).

148 We then performed isotope labelling experiments by adding $\left[\mathrm{U}-{ }^{13} \mathrm{C}_{5}\right]$-Gln to the culture medium. 149 HUVECs were then infected with Chlamydia and incubated in this medium for 36 hours. LC-MS 150 analysis revealed the labelling pattern of Gln, Glu, Asp and several TCA cycle metabolites (Fig. $1512 \mathrm{~d}$ ). We also observed evidence for reductive carboxylation of $\alpha-K G$ by the host cells as there 152 was a substantial amount of the $M+5$ isotopologue of citrate. In contrast, Gln-derived labelling 153 of pyruvate was almost absent (Fig. 2d), indicating that the enzymes of gluconeogenic pathway responsible for the conversion of oxaloacetate to pyruvate are inactive under these conditions.

155 Interestingly, while the labelled fraction of Gln did not change upon infection, the M+5 labelled 156 isotopologues of Glu and $\alpha-K G$ increased, indicating increased glutaminolysis in infected cells 157 (Fig. 2d). Moreover, the M+4 isotopologues of succinate, fumarate, malate and aspartate also 158 increased following Chlamydia infection, most likely due to enhanced entry of Gln-derived 159 carbons into the TCA cycle (Fig. 2d).

160 To analyse if Chlamydia obtain building blocks for DAP biosynthesis from the host cell, we 161 performed stable isotope labelling using $\left[\mathrm{U}-{ }^{13} \mathrm{C}_{5}\right]-\mathrm{G}$ In and extracted bacterial cell wall 162 components by acidic hydrolysis. LC-MS detection of DAP confirmed that glutamine-derived 163 carbons are indeed incorporated into bacterial biomolecules essential for Chlamydia 164 proliferation (Fig. 2e,f). Moreover, the high proportion of the M+4 isotopologue in DAP suggests 165 that Chlamydia either takes up glutamine directly from the host cells and uses it to produce Asp 166 via its truncated TCA cycle, or that glutamine is first converted by the host cell into metabolic 167 intermediates that contain four ${ }^{13} \mathrm{C}$ carbon atoms (i.e. succinate, fumarate, malate, oxaloacetate 168 or Asp), which are then taken up by Chlamydia and used for the synthesis of DAP (Fig. 2d-f, 169 diagram in Fig. 2e). Moreover, the high abundance of the $M+1, M+2$ and $M+3$ isotopologues in 
170 DAP also indicates that Chlamydia uses metabolic intermediates that are formed by the

171 complete TCA cycle of the host cell. Production of these isotopologues depends on the second 172 and third round of the TCA cycle, which requires the activity of citrate synthase, aconitase and 173 isocitrate dehydrogenase present only in the host metabolism (see diagram in Extended data 174 Fig. 2). Together, these results clearly demonstrate that Chlamydia-infected cells increase 175 uptake and metabolism of several amino acids, particularly Gln which provides the metabolic 176 intermediates required for bacterial peptidoglycan production.

\section{Chlamydia infection increases c-Myc levels}

178 We next focused on how infected cells compensate for the increased Gln demand. RNA-seq 179 analysis and Gene Set Enrichment Analysis (GSEA, see Methods) including subsequent 180 validation by qPCR revealed upregulation of MYC target genes (Hallmark_MYC_Targets_V2) 181 in cells infected with Chlamydia (Fig. 3a; Extended data Fig. 3a). The proto-oncogene c-MYC is 182 also known as a 'master regulator' of cellular metabolism ${ }^{11}$, in particular mitochondrial glutamine 183 metabolism ${ }^{12,13}$. In agreement with previous data ${ }^{14}$, c-Myc levels were strongly increased 184 already 12 hours post infection (hpi) and remained elevated up to $36 \mathrm{hpi}$ in primary epithelial 185 cells from human or mouse fimbriae (Fimb cells) (Extended data Fig. 3b,c), and human 186 osteosarcoma U2OS cells (Extended data Fig. 3d). Similar results were obtained upon infection 187 with different Chlamydia species (Extended data Fig. 3e-g).

188 Further investigation unveiled a transient upregulation of c-Myc mRNA expression in response 189 to Chlamydia infection (Extended data Fig. 3h) and the accumulation of c-Myc protein in the 190 nucleus (Extended data Fig. 3i,j). In addition, c-Myc was phosphorylated at the conserved 191 residues serine 62 (S62) and threonine 58 (T58) (Fig. 3b) involved in the control of c-Myc protein 192 stability ${ }^{15}$ in response to mitogenic signalling ${ }^{16}$ and Chlamydia infection ${ }^{14}$. Indeed, we found 193 markedly reduced levels of ubiquitinated c-Myc (Fig. 3c) and stabilized c-Myc in infected cells 194 with inhibited translation (Fig. 3d), demonstrating that c-Myc is really stabilized upon infection. 195 
197 MAPK and PI3K pathways activated during infection are both critical for chlamydial development

198 17-19. The same pathways have been demonstrated to function in the stabilization of c-Myc (Fig. 1993 3e ${ }^{20}$. Inhibition of MAPK or PI3K pathways using specific inhibitors indeed prevented the up200 regulation of c-Myc protein levels and attenuated the propagation of the bacteria in infected cells 201 (Fig. 3f,g). Interestingly, the downstream effector GSK3ß (Fig. 3e) was phosphorylated at S9 in 202 infected cells (Fig. 3h,i), a modification known to inhibit glycogen synthesis and to increase free 203 glucose in the cell 21.

204 Intriguingly, Chlamydia primary infection (Fig. 4a-c; AHT control in Extended data Fig. 4a) and 205 infectivity (Extended data Fig. 4b,c) was rescued in U2OSTet-On cells upon anhydrous tetracycline 206 (AHT)-induced expression of $\mathrm{c}-\mathrm{Myc}^{22}$ despite inhibition of the MAPKor PI3K pathway, indicating 207 that the anti-chlamydial activity of U0126 and Ly294002 is mediated by the down-regulation of 208 c-Myc. The central role of c-Myc for chlamydial growth was further supported by experiments 209 with the chemical c-Myc inhibitor 10058-F4 (Fig. 4d, Extended data Fig. 4d) and the silencing of 210 c-Myc expression (Fig. 4e,f; Extended data Fig. 4e,f).

\section{Chlamydia depends on host cell GIn uptake}

213 GSEA of the RNA-seq data also revealed a strong influence of Chlamydia infection on the host

214 metabolite transporter and cellular amino acid and amine metabolic pathway (Extended data 215 Fig. 5a-c). Gln turned out to be essential for the intracellular growth of Chlamydia, since they 216 failed to form inclusions and replicate in host cells cultured in medium without Gln (Fig. 5a,b and

217 Extended data Fig. 5d). Bacteria from these cultures did not initiate infections in fresh cells in 218 the presence of Gln, demonstrating that no infectious progeny were produced in the absence of 219 Gln (Fig. 5c,d, Extended data Fig. 5e). To investigate if Gln plays a role in EB to RB conversion 220 in host cells, we used the EB-RB reporter strain $\mathrm{Ct} m \mathrm{mCh}(\mathrm{GroL2}) \mathrm{GFP}(\mathrm{OmcAL} 2)^{23}$. In the 221 absence of GIn, Chlamydia, did not convert into the actively replicating RB form, with low GFP 222 expression and constant mCherry fluorescence (Fig. 5e).

223 c-Myc can function in a glutamine sensing pathway via a mechanism dependent on the 3' UTR 224 of the gene ${ }^{24}$. We used HCT116 cells expressing the MYC-ER fusion protein from a construct 
225 lacking the 3'UTR, which allows the restoration of c-Myc expression in Gln-deprived cells.

226 However, Gln depletion severely attenuated the growth and development of Chlamydia (Fig.

$2275 f, g)$, and even complementing with downstream metabolites like nucleosides did not 228 compensate for the lack of Gln (Extended data Fig. 5f), indicating that c-Myc cannot rescue 229 chlamydial growth in the absence of Gln.

Chlamydia infection reprograms host cell metabolism by inducing glutamine uptake and

\section{2 catabolism}

233 The data obtained so far suggested that Gln is also a central host-derived amino acid 234 metabolised by fast replicating Chlamydia. We therefore investigated the regulation of genes 235 related to glutamine uptake and catabolism. These analyses revealed SLC1A5/ASCT2 ${ }^{25}$ among 236 the top upregulated genes during infection (Fig. 6a). SLC1A5/ASCT2 and glutaminase (GLS1), 237 one of the enzymes converting Gln to Glu (Fig. 6b), have been shown to be c-Myc target genes 238 induced to enhance glutamine uptake and catabolism in cancer cells ${ }^{12,26}$.

239 Western blots of control and Chlamydia-infected samples revealed a 5.2-fold up-regulation of 240 SLC1A5 and a 1.8-fold up-regulation of GLS1 (Fig. 6c). Induction of SLC1A5 depended on 241 viable bacteria since heat-killed Chlamydia neither induced c-Myc nor SLC1A5 (Extended data 242 Fig. 6a). Furthermore, depletion of SLC1A5 or GLS1 (Fig. 6d,e) or chemical inhibition of SLC1A5 243 or GLS1 using GPNA and BPTES (Fig. 6f,g) drastically reduced chlamydial replication. Since 244 SLC1A5 and GLS1 are transcriptional targets of c-Myc ${ }^{12,27}$, we knocked down c-Myc with 245 siRNA, which resulted in strong depletion of both proteins and also blocked chlamydial growth 246 (Fig. 6h).

247 Most interestingly, when we depleted c-Myc in cells overexpressing SLC1A5 we could retain 248 Chlamydia growth during the primary infection, but the progeny was not infectious (Fig. 6i and 249 Extended data Fig. 6b), in line with a role of Gln in establishing the primary infection (Fig. 1e). 250 However, other c-Myc regulated host cell functions, for example glutaminolysis and the 251 production of TCA cycle intermediates, may be essential for the full development of Chlamydia. 
252 Taken together, these data demonstrate the importance of an infection-dependent regulation of

253 C-Myc and its targets SLC1A5 and GLS1 in the reprogramming of the Gln metabolism in host 254 cells to promote Chlamydia replication and development.

255 Recently, Slc1a5knockout mice have been generated which show no major phenotype ${ }^{28}$. Mass 256 spectrometric analysis revealed that organoids generated from fallopian tube tissue from these 257 mice contain almost $50 \%$ lower levels of Gln and the downstream metabolites Glu, Asp and Ala 258 compared to organoids from wild type mice (Extended data Fig. 6c). Interestingly, trans-cervical 259 infection of these mice revealed a significant growth defect of $C$. trachomatis and the mouse 260 pathogenic strain C. muridarum in the absence of Slc1a5 (Fig. 6j,k). Moreover, infection and 261 progeny formation were considerably reduced in fallopian tube organoids developed from 262 Slc1a5 KO mice (Fig. 6I, Extended data Fig. 6d), demonstrating that cells of S/c1a5 KO mice 263 have reduced capacity to allow chlamydial replication. Further experiments are required to 264 demonstrate that the strong phenotype observed on chlamydial growth in these organoids 265 depends on disturbed reprogramming of glutamine metabolism. These data suggest that limiting 266 the uptake of the non-essential amino acid glutamine could serve as a therapeutic approach 267 against Chlamydia infection.

\section{Discussion}

269 After the completion of the developmental cycle EBs released from the host cell have to avoid 270 the transition to RBs since only EBs are capable of entering uninfected host cells and initiate a 271 new generation of progeny. The longstanding view that EBs are metabolically inactive has 272 recently been revised, since it was found that EBs kept in axenic medium in the presence of 273 G6P, a metabolite available to Chlamydia only inside host cells, are able to generate ATP via 274 glycolysis ${ }^{7}$. However, despite this active metabolism, EBs do not initiate the conversion to RBs 275 under these conditions. Here we provide evidence that GIn is the key metabolite that initiates 276 peptidoglycan synthesis and the EB to RB transition. While the concentration of Gln in uterine 277 fluid is as low as $0.13 \mathrm{mM}^{29}$, in the cytoplasm of mammalian cells Gln can reach levels of 2 to $27830 \mathrm{mM}^{30}$. The strong differences in GIn concentrations between the outside and the inside of 279 host cells strongly support a function of Gln as a metabolic trigger for EB to RB transition. 
280 Our finding that L-glutamine serves as a crucial amino acid for the replication of Chlamydia 281 corroborates previous findings ${ }^{31,32}$. Many of the glutamine-derived intermediates in Chlamydia 282 serve as precursors for peptidoglycan biosynthesis (Fig. 1d and Extended data Fig. 1b) 283 underlining the central role of glutamine for the chlamydial metabolism. Chlamydiae do not form 284 a peptidoglycan sacculus typical for other Gram-negative bacteria but only assemble 285 peptidoglycan rings in the mid-cell of actively dividing RBs. We detected the accumulation of 286 peptidoglycan here also in Chlamydia outside of host cells but only in the presence of Gln (Fig. $2871 \mathrm{f}, \mathrm{g}$ ), where we also observed a change in the morphology of the EBs (Fig. 1e, Extended data 288 Fig. 1e). Peptidoglycan accumulated in or close to the bacteria and was not assembled as a ring 289 which could indicate that the crosslinking of the peptidoglycan disaccharide pentapeptide did 290 not occur in this setup. The transcriptional activation of early genes and the increase in the copy 291 number of the bacterial genomes (Fig. 1 h,i), however, indicated that Chlamydia already initiate 292 the start of the replication machinery.

293 Chlamydia and other obligate intracellular bacteria replicate in differentiated cells that run the 294 reduced metabolism of non-dividing cells. Since all metabolites of replicating Chlamydia 295 originate from the host cell, the metabolism of the cell must drastically change to meet the 296 requirements of the infection. Glutamine is one of the most abundant amino acids in serum and 297 fast-growing cells take up glutamine to support anabolic metabolism at multiple nodes. We show 298 here that Chlamydia depend on the direct uptake of glutamine as well as on glutamine-derived 299 host metabolites and therefore reprograms the host cell by stabilizing the central metabolism 300 regulator $\mathrm{c}-\mathrm{Myc}$. The profile of $\mathrm{c}-\mathrm{Myc}$ regulated genes and the dependence of the replicating 301 Chlamydia on glutaminolysis parallels the reprogramming of dormant cells to fast proliferating 302 and in particular tumour cells ${ }^{33}$. c-Myc promotes glutamine uptake and glutaminolysis by 303 increasing the expression of the glutamine transporters ASCT2/SLC1A5 and SNAT5/SN2 and 304 of glutaminase (GLS) ${ }^{13}$. We demonstrate here that bacteria induce host cell glutamine uptake 305 and glutaminolysis for their replication. In addition, chlamydial growth was severely affected in 306 genital infections of ASCT2/SLC1A5 knockout mice and is thereby the demonstration of a 307 dominant role of host cell glutamine reprogramming in an in vivo infection. Gln is channelled into 
308 different anabolic and catabolic pathways via glutaminolysis, generating Glu and subsequently

309 a-KG. While GIn-derived metabolic intermediates have been shown to be essential for the

310 infection and replication of several viruses ${ }^{34-39}$, the relevance of glutamine metabolism for viral

311 infection still has to be demonstrated in vivo.

312 In addition to glutamine transporters and glutaminase, we found other prominent c-Myc313 regulated amino acid transporters like SLC43A1, SLC7A11, and SLC7A1 are also upregulated 314 during infection (Fig. 6a). This global reprogramming of amino acid supply in infected host cells

315 by c-Myc stabilization may explain our intriguing finding that overexpression of SLC1A5 can only 316 partially rescue chlamydial infection in c-Myc-depleted cells. While SLC1A5 restores bacterial 317 replication, it fails to support the production of infectious progeny in the absence of c-MYC. Thus, 318 the provision of glutamine is essential, but not sufficient to permit the complex cycle of 319 chlamydial replication and development.

320 The central addiction of Chlamydia to host glutamine is reminiscent of mammalian cell 321 proliferation, malignant transformation and therefore a hallmark of cancer cells which use 322 extracellular glutamine to fulfil the metabolic demands of producing cell mass. Glutamine 323 addicted tumour cells and, as we show here, Chlamydia-infected cells are therefore highly 324 sensitive to pharmacological disruption of glutamine metabolism. Current approaches to target 325 c-Myc or glutamine metabolism as targets for innovative cancer therapy may also prove to be 326 efficient in treating Chlamydiainfection.

\section{Methods}

\section{Chlamydia strains used in the study}

330 Chlamydia trachomatis (serovar $\mathrm{L}_{2} / 434 / \mathrm{Bu}$ and $\mathrm{D}$ ) were used in this study. Some experiments 331 were also performed using Chlamydia muridarum and Chlamydia pneumoniae. Chlamydia were 332 prepared as previously published. Briefly, Chlamydia were grown in HeLa229 cells 333 (ATCC ${ }^{\circledR}$ CCL2. $1^{\text {TM}}$ ) at an MOI (multiplicity of infection) of 1 for 48 hours $(\mathrm{h})$. The cells were lysed 334 using glass beads $(15 \mathrm{~mm})$ for 3 minutes $(\mathrm{min})$ and centrifuged at $2,000 \mathrm{~g}$ for $10 \mathrm{~min}$ to remove 335 the cell debris. The supernatant containing bacteria was collected and centrifuged at $24,000 \mathrm{~g}$ 
336 for $30 \mathrm{~min}$ at $4^{\circ} \mathrm{C}$. The pellet was washed and resuspended in SPG buffer $(0.25 \mathrm{M}$ sucrose/10

$337 \mathrm{mM}$ sodium phosphate $/ 5 \mathrm{mM}$ glutamic acid), aliquoted and stored at $-80^{\circ} \mathrm{C}$. Chlamydia EBs and 338 cell lines used in the study were verified to be free of Mycoplasma contamination via PCR. The 339 bacteria were titrated and were used at an $\mathrm{MOI}$ of 1 in all experiments unless other stated. After 3401 hpi the media was replaced with fresh RPMI containing 5\% FCS, infected cells were cultured 341 at $37^{\circ} \mathrm{C}$ and $5 \% \mathrm{CO}_{2}$.

\section{Culture of C. trachomatis in axenic medium}

343 Chlamydia trachomatis L2 was propagated in HeLa229 cells, isolated, purified and incubated in 344 different axenic media as previously described ${ }^{6}$. In brief, HeLa229 cells were seeded in T175 345 flasks and infected at an MOI of one. Forty-eight hours post infection, cells were scraped off, 346 disrupted with glass beads and EBs were purified using 60-20\% Renografin gradient (Meglumin 347 diatrizoate (Sigma-Aldrich M5266), Sodium diatrizoate hydrate (Sigma-Aldrich S4506), Sodium 348 citrate hydrate (Applichem A4522), EDTA (Servca 11280) add to $50 \mathrm{ml} \mathrm{HBSS}$ (Gibco 14025$349050) \mathrm{pH}-7.4$, sterile filtered in $0.2 \mu \mathrm{m}$ filter and stored at $\left.4^{\circ} \mathrm{C}\right)$. EBs were resuspended in axenic 350 media (basic DMEM (Sigma-Aldrich) supplemented with sodium bicarbonate (44 mM), phenol 351 red $(42 \mu \mathrm{M})$ and glucose-6-phosphate $(0.5 \mathrm{mM})$ and incubated for respective time points at

$35237^{\circ} \mathrm{C}$. According to the experimental set-up, $1 \mathrm{mM}$ of L-glutamine / L-glutamate / pyruvate / $\alpha$ 353 ketoglutarate / [U- $\left.{ }^{13} \mathrm{C}_{5}\right]$ glutamate / [U- $\left.{ }^{13} \mathrm{C}_{5}\right]$ glutamine / $\left[{ }^{15} \mathrm{~N}\right.$-amine $]$ glutamine or $\left[{ }^{15} \mathrm{~N}\right.$-amide] 354 glutamine was added to the media. After incubation, samples were centrifuged for 30 min at $35521,500 \mathrm{~g}$ at $4^{\circ} \mathrm{C}$ and supernatant was transferred to a new tube. Supernatant and pellets were 356 heat-inactivated $\left(10 \mathrm{~min}\right.$ at $\left.90^{\circ} \mathrm{C}\right)$ and stored at $-80^{\circ} \mathrm{C}$ for further analysis.

\section{Substrate uptake analysis from axenic culture}

\section{8 a. $\alpha$-ketoglutarate:}

$3590.2 \mathrm{ml}$ of the supernatant from the axenic medium was spiked with $20 \mu \mathrm{l}$ of a $5 \mathrm{mM}$ norvaline 360 solution (internal standard) and dried under $\mathrm{N}_{2}$ flux. The residue was treated with $50 \mu \mathrm{l}$ 361 methoxyamine in pyridine $(20 \mathrm{mg} / \mathrm{ml})$ at $40^{\circ} \mathrm{C}$ for $90 \mathrm{~min}$, and subsequently with $50 \mu \mathrm{l}-($ tert362 butyldimethylsilyl)- $N$-methyl trifluoroacetamide containing $1 \%$ tert-butyldimethylsilyl chloride (MTBSTFA) at $70^{\circ} \mathrm{C}$ for $30 \mathrm{~min}$. This solution was taken for analysis. 


\section{b. Glutamate and glutamine}

$3650.1 \mathrm{ml}$ of the supernatant from the axenic medium containing $\left[\mathrm{U}^{-13} \mathrm{C}_{5}\right]$ glutamate or $\left[\mathrm{U}-{ }^{13} \mathrm{C}_{5}\right]$ 366 glutamine was spiked with $20 \mu \mathrm{l}$ of a $5 \mathrm{mM}$ non-labelled glutamate or glutamine solution (internal

367 standard) and dried under $\mathrm{N}_{2}$ flux. The residue was treated with $50 \mu$ acetonitrile and $50 \mu \mathrm{l}$ 368 MTBSTFA at $70^{\circ} \mathrm{C}$ for 30 min and taken for analysis.

\section{Isotopologue profiling with Chlamydia from axenic culture:}

370 Bacterial pellets were suspended in $1 \mathrm{ml}$ of methanol and were mechanically disrupted using a 371 ribolyser $(3 \times 20 \sec 6.5 \mathrm{~m} / \mathrm{s})$. Afterwards the solution was centrifuged (10,000 $\mathrm{g}$ for $20 \mathrm{~min}$, $3724^{\circ} \mathrm{C}$ ). This procedure was performed twice. The supernatants were combined and then dried 373 under $\mathrm{N}_{2}$ flux. The residue was treated with $50 \mu \mathrm{l}$ of MTBSTFA and $50 \mu \mathrm{l}$ of water free acetonitrile 374 at $70^{\circ} \mathrm{C}$ for $30 \mathrm{~min}$. The tert-butyldimethylsilyl (TBDMS)-derivatives of amino acids and other 375 metabolites were then analysed by GC/MS. The residual cell debris after centrifugation was 376 subjected to acidic hydrolysis as described earlier ${ }^{40}$ and protein bound amino acids as well as 377 diaminopimelate (DAP; retention time, $24.48 \mathrm{~min} ; \mathrm{m} / \mathrm{z} 589$ ) were analysed as TBDMS 378 derivatives.

\section{GC/MS conditions}

380 All derivatives mentioned above were analysed by GC-MS using a GCMS-QP 2010 Ultra 381 spectrometer (Shimadzu, Duisburg, Germany) equipped with a EquityTM -5, fused silica capillary 382 column, $30 \mathrm{~m} \times 0.25 \mathrm{~mm} \times 0.25 \mu \mathrm{m}$ film thickness. All data were collected using LabSolution 383 software (Shimadzu). The samples were analysed three times as technical replicates. The 384 overall ${ }^{13} \mathrm{C}$ excess (mol-\%) and the relative contributions of isotopomers (\%) were computed by 385 an Excel-based in-house software package according to published procedures ${ }^{41}$.

\section{TBDMS-derivatives of polar metabolite mixtures}

387 The column was first developed at $100^{\circ} \mathrm{C}$ for $2 \mathrm{~min}$, then using a gradient of $3^{\circ} \mathrm{C} \mathrm{min}^{-1}$ to $234^{\circ} \mathrm{C}$, 388 followed by $1^{\circ} \mathrm{C} \min ^{-1}$ to $237^{\circ} \mathrm{C}$ and $3^{\circ} \mathrm{C} \min ^{-1}$ to $260^{\circ} \mathrm{C}$. Finally, the column was heated at a 389 gradient of $10^{\circ} \mathrm{C} \mathrm{min}-1$ to a final temperature of $320^{\circ} \mathrm{C}$ where it was hold for $2 \mathrm{~min}$. 
391 The column was first developed at $150^{\circ} \mathrm{C}$ for $3 \mathrm{~min}$, then using a gradient of $7^{\circ} \mathrm{C} \mathrm{min}-1$ to $280^{\circ} \mathrm{C}$

392 where it was hold for 5 min. For quantitative DAP analysis in Chlamydia DAP DL-2,6-

393 Diaminopimelic acid (Sigma Aldrich No. 92591) was used as standard for the calibration.

394 Chlamydial DAP synthesis in axenic media was performed by an approximate quantification of

395 DAP on the basis of peak integrals of the samples used for isotopologue profiling with ${ }^{13} \mathrm{C}_{5}-\mathrm{Gln}_{\mathrm{n}}$

396 (Fig. 1d). This analysis revealed that the preparation contained approximately $0.1 \mathrm{mg}$ DAP per

397 gram wetweight of Chlamydia. The newly built DAP is highly labelled and the overall ${ }^{13} \mathrm{C}$ excess

398 of $40 \%$ shown as a mixture of newly built ${ }^{13} \mathrm{C}$ labelled DAP and residual unlabelled DAP from

399 the inoculum. On the basis of $40 \%$ newly formed DAP, we could estimate a value of about 0.04

$400 \mathrm{mg}$ DAP formation per gram cells of Chlamydia in the presence of GIn in axenic medium

401 (Extended data Fig. 1c).

402 Transmission electron microscopy

403 Chlamydial EBs were incubated with the axenic medium with G6P and with or without GIn. The

404 bacteria pellet was fixed with $2.5 \%$ glutaraldehyde (50 mM sodium cacodylate (pH 7.2), $50 \mathrm{mM}$

$405 \mathrm{KCl}, 2.5 \mathrm{mM} \mathrm{MgCl}_{2}$ ) at room temperature. The cells were incubated for $2 \mathrm{~h}$ at $4^{\circ} \mathrm{C}$ with $2 \%$

$406 \mathrm{OsO}_{4}$ buffered with $50 \mathrm{mM}$ sodium cacodylate $(\mathrm{pH} 7.2)$, washed with distilled $\mathrm{H}_{2} \mathrm{O}$ and

407 incubated overnight at $4^{\circ} \mathrm{C}$ with $0.5 \%$ uranyl acetate (in distilled $\mathrm{H}_{2} \mathrm{O}$ ). The cells were 408 dehydrated, embedded in Epon812 and ultrathin-sectioned at $50 \mathrm{~nm}$. Sections were stained 409 with $2 \%$ uranyl acetate in ethanol followed by staining with lead citrate and analysed in a 410 Zeiss EM10 microscope (Zeiss). Electron micrographs were processed using ImageJ (Fiji).

\section{Click chemistry and FACS analysis of chlamydial EBs}

412 Click chemistry was performed as described in ${ }^{2}$. Axenic culture with purified EBs from 413 Chlamydia was fed with ADA-DA $(10 \mu \mathrm{M})$. Clickable Alexa Fluor 532-azide and Click-iT® Cell 414 Reaction Buffer Kit were purchased from Invitrogen and fixed with 4\% PFA. The bacteria were 415 stained with cHsp60 antibody and subjected to microscopy or further analysed by FACS 416 (Accuri).

\section{Copy number of Chlamydia genomes in axenic culture}


418 Chlamydia were grown in HeLa229 for $48 \mathrm{~h}$. The cells were lysed and the EBs were purified by

419 renografin gradient separation as explained above. The bacteria were pooled by centrifugation

420 and re-suspended in axenic medium without Gln. The re-suspended Chlamydia were split into

421 two aliquots and Gln was added into one of them and incubated at $35^{\circ} \mathrm{C}$ for $24 \mathrm{~h}$. The bacteria 422 were further pelleted and DNA was isolated using DNAzol reagent (Thermo Fisher Scientific).

423 Quantitative PCR was used to enumerate Chlamydia genome copy number. The following 424 primers were used for amplifying the $C$. trachomatis lytA gene that was cloned into the vector:

425 forward primer 5'-TCTAAAGCGTCTGGTGAAAGCT-3' and reverse primer 5'426 GAAATAGCGTAGTAATAATACCCG-3'. Data were analysed by using the Step One Plus 427 software package (Applied Biosystems). GraphPad Prism 7 was used to generate the graph.

428 Cell culture and transfection

429 HeLa229 were used for propagating bacteria and for basic experiments. Epithelial cells isolated 430 from human fimbriae (Fimb cells), U2OS (ATCC ${ }^{\circledR}$ HTB-96 $^{\text {TM }}$ ), HCT116 $\left(\right.$ ATCC $^{\circledR}$ CRL-247 ${ }^{\text {TM }}$ ) and 431 HUVECs $\left(\right.$ ATCC ${ }^{\circledR}$ CRL-1730 ${ }^{\text {TM }}$ ) were also used in the study. HUVECs were used in the high 432 throughput RNA sequencing. All cell lines were tested negative for mycoplasma contamination 433 via PCR. HeLa229 and human Fimb cells were grown in RPMI1640 + GlutaMAXTM (Gibco TM $43472400-054)$ with $10 \%$ heat inactivated FCS (Sigma-Aldrich F7524). U2OS and HCT116 cells 435 were cultured in DMEM (Sigma-Aldrich D6429) with 10\% heat inactivated FCS. HUVECs were 436 cultured in Medium 200 (Gibco ${ }^{\text {TM }}$ M200500) containing 1x LSGS (Gibco ${ }^{\text {TM }}$ S00310). For 437 glutamine deprivation experiments, the cells were first seeded in RPMI1640 + GlutaMAX ${ }^{\mathrm{TM}}$ or 438 DMEM, high glucose (Sigma-Aldrich D6429). The following day the medium was changed to the 439 basic formulation of DMEM (Sigma-Aldrich D5030) supplemented with $5 \%$ dialyzed FCS 440 (Sigma-Aldrich F0392), 1 or $4.5 \mathrm{~g} / \mathrm{I}$ D-glucose (for HeLa229/ U2OS or human Fimb respectively) 441 and varying concentrations of L-glutamine (according to experimental setup). All cell lines 442 obtained from ATCC were authenticated by the company.

443 Cells were transfected with plasmid DNA at a confluency of $60 \%$ with Polyethylenimine (PEI) or 444 X-treme GENETM HP DNA transfection reagent (Roche) and OptiMEM transfection medium 445 (Gibco) in 5\% FCS medium. After $5 \mathrm{~h}$, transfection medium was replaced by fresh RPMI 
446 supplemented with 5\% FCS medium. The plasmids used in the study are described in the

447 Extended data file. siRNA against SLC1A5 (sc-60210) and c-Myc (sc-29226) was obtained from

448 Santa cruz Biotech.

449 Metabolic profiling

450 For this study HUVECs were seeded in triplicates, either uninfected or infected with $C$. 451 trachomatis serovar L2 for 36 hours. After the respective time medium was collected, snap 452 frozen in liquid nitrogen, and the cells were washed with ice cold $154 \mathrm{mM}$ ammonium acetate 453 (Sigma) and snap frozen in liquid nitrogen. The cells were harvested after adding $480 \mu \mathrm{l}$ cold $454 \mathrm{MeOH} / \mathrm{H}_{2} \mathrm{O}(80 / 20, \mathrm{v} / \mathrm{v})$ (Merck) to each sample containing Lamivudine (Sigma) standard (10 $455 \mu \mathrm{M}$ ). The cell suspension was collected by centrifugation and transferred to an activated (by 456 elution of $1 \mathrm{ml} \mathrm{CH}{ }_{3} \mathrm{CN}$ (Merck)) and equilibrated (by elution of $1 \mathrm{ml} \mathrm{MeOH} / \mathrm{H}_{2} \mathrm{O}(80 / 20, \mathrm{v} / \mathrm{v})$ ) 457 RP18 SPE-column (Phenomenex). The eluate was collected and evaporated in a SpeedVac 458 concentrator. The residue was dissolved in $50 \mu \mathrm{L}$ of $5 \mathrm{mM} \mathrm{NH} 4 \mathrm{OAc}$ in $\mathrm{CH} 3 \mathrm{CN} / \mathrm{H} 2 \mathrm{O}(25 / 75)$.

459 Each sample was diluted 1:2 (cells) or 1:5 (medium) in $\mathrm{CH} 3 \mathrm{CN} .5 \mu \mathrm{l}$ of sample was applied to 460 HILIC column (Acclaim Mixed-Mode HILIC- 1,3 $3 \mathrm{~m}, 2.1^{*} 150 \mathrm{~mm}$ ). Metabolites were separated 461 at $30^{\circ} \mathrm{C}$ by LC using a DIONEX Ultimate 3000 UPLC system (Solvent A: $5 \mathrm{mM} \mathrm{NH} 4 \mathrm{OAc}$ in $462 \mathrm{CH}_{3} \mathrm{CN} / \mathrm{H}_{2} \mathrm{O}$ (5/95), Solvent B: 5 mM NH${ }_{4} \mathrm{OAc}$ in $\mathrm{CH}_{3} \mathrm{CN} / \mathrm{H}_{2} \mathrm{O}$ (95/5); Gradient: linear from 100\% 463 B to $50 \%$ B in 6 min, followed by 15 min const. $40 \%$ B). MS-Analysis was done on a Thermo 464 Scientific QExactive instrument in alternating positive and negative mode. Peak determination 465 and semi-quantitation were performed using TraceFinder ${ }^{\mathrm{TM}}$ Software. For determination of 466 protein content forthe datanormalization, BCA assay (Thermo Fisher Scientific) was performed. 467 The pellet of the cell samples was dried, resuspended in $0.2 \mathrm{M}$ sodium hydroxide (Roth), boiled 468 for $20 \mathrm{~min}$ at $95^{\circ} \mathrm{C}$ and absorbance was measured at $550 \mathrm{~nm}$. Prism GraphPad was used for 469 statistical analysis.

\section{Western blotting and antibodies}

471 Lysates for Western blot analysis were prepared by directly lysing cells in SDS sample buffer 472 (62.5 mM Tris, pH 6.8, 2\% SDS, 20\% glycerol and 5\% ß-mercaptoethanol) in ice. Western blot 473 analysis was performed as described ${ }^{42}$. Briefly protein samples were separated in the $6-12 \%$ 
474 SDS-PAGE (Peqlab) and transferred to a PVDF membrane (Roche) in a semidry electroblotter

475 (Thermo Fisher Scientific). The membrane was further blocked in tris buffer saline containing $476 \quad 0.05 \%$ Tween20 and 5\% bovine serum albumin or dry milk powder. The primary antibody 477 against c-Myc (Y69: ab-32072), pc-Myc Thr58 (ab-28842) and pc-Myc Ser62 (ab-51156) 478 SLC1A5 (ab-84903) glutaminase (ab-156876) was purchased from Abcam. The T-ERK (cs479 9180), pERK (cs-9106), T-AKT (cs-9272), pAKT Ser473 (cs-9271), were obtained from Cell 480 Signaling. Chlamydial HSP60 (sc-57840) and anti-ubiquitin (sc-8017) antibody was purchased 481 from Santa Cruz Bioscience and B Actin antibody from Sigma (A5441). Proteins were detected 482 with secondary antibodies coupled with HPR (Santa Cruz Bioscience) using ECL system 483 (Pierce) and Intas Chem HR 16-3200 reader. Quantification of blots was done by FIJI (ImageJ) 484 software.

485 Immunoprecipitation

486 Uninfected and Chlamydia-infected (MOI 1) HeLa229 cells were lysed using denaturing buffer 487 (RIPA lysis buffer: 50mM Tris-HClpH-7.5, 150mM NaCl, 1\% Triton-X100, 1\%NP-40, 0.1\%SDS, $48810 \%$ glycerol containing Complete protease inhibitor cocktail (Roche) and MG-132, proteasome 489 inhibitor) to prevent co-precipitation of interacting partners of c-Myc. Lysates from $7 \times 10^{6}$ cells 490 were prepared as described before and incubated with $3 \mu \mathrm{g}$ anti-c-Myc antibody for $1 \mathrm{~h}$ at $4^{\circ} \mathrm{C}$ 491 followed by incubation with protein G magnetic beads (Dynabeads, Thermo Fisher Scientific) 492 for $2 \mathrm{~h}$ at $4^{\circ} \mathrm{C}$. The samples were washed several times and eluted by addition of $2 x$ SDS-sample 493 buffer and heating to $94^{\circ} \mathrm{C}$. Samples were separated with SDS-PAGE and visualized by 494 immunoblotting after probing against anti ubiquitin antibody.

\section{Nuclear-cytoplasmic isolation}

496 HeLa229 cells were plated in $150 \mathrm{~mm}$ dishes and either left uninfected or infected with 497 Chlamydia (MOI 1) for the mentioned period of time. The cells were washed with ice cold PBS. 498 The cells were scraped into a falcon. The cells were centrifuged and resuspended in buffer 499 containing 10 mM Hepes-KOH pH 7.9, 10 mM KCl, 1.5 mM MgCl , 0.5 mM DTT, 0.05\% NP-40, 500 protease inhibitors and incubated for $20 \mathrm{~min}$ in ice. The cells were then homogenized with a 501 dounce homogenizer (10 strokes). The cells were centrifuged at $4,000 \mathrm{RPM}$ for 5 min at $4^{\circ} \mathrm{C}$, 
502 the supernatant containing cytoplasmic proteins was collected and lysed with 2x SDS-sample

503 buffer. The pellet was resuspended in buffer containing 20 mM Hepes- $\mathrm{KOH} \mathrm{pH} \mathrm{7.9,} 400 \mathrm{mM}$

$504 \mathrm{NaCl}, 1.5 \mathrm{mM} \mathrm{MgCl}$, $0.2 \mathrm{mM}$ EDTA, 15\% glycerol, $0.5 \mathrm{mM}$ DTT, protease inhibitors and

505 incubated for another 20 min and vortexed. Further the sample was centrifuged at 14000 RPM

506 for $10 \mathrm{~min}$ at $4^{\circ} \mathrm{C}$, the pellet containing the nuclear extract was lysed in $2 x$ SDS-sample buffer

507 and analysed using Western blot.

508 Immunofluorescence analysis

509 The immunostaining was performed as described earlier. Briefly, HeLa229 and HUVEC cells

510 were grown on cover slips and infected with the indicated C. trachomatis strain at an MOI 1 for

511 indicated time points. The cells were washed with PBS, fixed with 4\% PFA/Sucrose and

512 permeabilized with $0.2 \%$ Triton-X-100/PBS for 30 min. Samples were blocked with $2 \%$ FCS/PBS

513 for $1 \mathrm{~h}$. All primary antibodies were incubated for $1 \mathrm{~h}$ at room temperature. Primary antibodies

514 were used in the following dilutions in 2\% FCS/PBS: anti-Hsp60 (1:500), c-Myc (1:200).

515 Samples were washed three times and incubated with a Cy2-/Cy3-/Cy5-conjugated secondary

516 antibody for $1 \mathrm{~h}$ in the dark. The cells were mounted on microscopic slide using Mowiol. Slides

517 were air dried for at least $24 \mathrm{~h}$ and examined using Leica DM2500 fluorescence microscope,

518 the images were analysed using LAS AF and Image J software.

\section{Inhibitor studies}

520 Hela229 cells were grown in the RPMI1640 +GlutaMAXTM or in the basic DMEM medium 521 supplemented with $5 \%$ dialysed FCS and $1 \mathrm{~g} / \mathrm{l}$ of D-Glucose and $4 \mathrm{mM} \mathrm{L}$-glutamine. The cells 522 were treated with $30 \mu \mathrm{M}$ MAPK inhibitor U0126 (Cell Signaling Technology), $30 \mu \mathrm{M}$ PI3K 523 inhibitor LY297004 (Cell Signaling Technology), the cell-permeable thiazolidinone c-Myc 524 inhibitor 10058-F4 (Sigma-Aldrich), $5 \mu \mathrm{M}$ glutaminase inhibitor BPTES (Sigma-Aldrich) or the 5 525 mM SLC1A5 inhibitor GPNA (Sigma-Aldrich) for the respective period of time. In general, cells 526 were infected with Chlamydia at an MOI 1 for different time points. The vehicle used for each 527 inhibitor was used in the appropriate concentration. The cells were lysed and analysed using 528 Western blotting.

\section{RNA Sequencing and NGS Data Analysis}


530 Differential gene expression from infected host cells: Total RNA was isolated from

531 uninfected or infected cells (12, 24 and $36 \mathrm{~h}$ post infection) and was used for RNA sequencing

532 (RNA seq). Libraries for RNA Seq were generated using NEBNext ${ }^{\circledR}$ Ultra ${ }^{\mathrm{TM}}$ RNA Library Prep

533 Kit for Illumina® with 12 PCR cycles for amplif ication and sequenced on Illumina NextSeq500

534 platform. FASTQ generation was carried out using CASAVA and the quality check was

535 performed using FastQC. Reads from FASTQ files were aligned to hg19 genome using Bowtie2

536 and differential gene regulation calculation was carried out based on edgeR algorithm. The gene

537 expression changes between infected and uninfected cells were normalized to library size were

538 used for carrying out Gene Set Enrichment Analysis (GSEA) using weighted parameters on C2,

539 C5 and Hallmark gene sets downloaded from MSigDB database ${ }^{43}$. In this analysis, the nominal

$540 \mathrm{p}$-value is calculated using an empirical phenotype-based permutation test procedure. The

541 permutation-based false-discovery rate (FDR) $Q$ value is generated by correcting for gene set

542 size and multiple hypothesis testing. Heat map for Fig 6a was generated using heat map

543 package in $\mathrm{R}$ with default settings.

544 Differential gene expression of Chlamydia in axenic culture: Renografin purified Chlamydia 545 were cultured either in DMEM media (without Glucose/G6P without GIn) or in DMEM with only 546 G6P or with DMEM with G6P and Gln for $12 \mathrm{~h}$ at $35^{\circ} \mathrm{C}$. The bacteria were pelleted by 547 centrifugation at $12,000 \mathrm{~g}$ for $10 \mathrm{~min}$ at $4^{\circ} \mathrm{C}$. Total RNA was isolated from the bacteria and was 548 used for RNA sequencing (RNA-seq). Libraries for RNA-seq were generated using 549 NEBNext ${ }^{\circledR}$ Ultra ${ }^{\mathrm{TM}}$ RNA Library Prep Kit for Illumina $\AA^{\circledR}$ with 12 PCR cycles for amplification and 550 sequenced on Illumina NextSeq500 platform. FASTQ generation was carried out using GALAXY 551 platform (DKFZ, Heidelberg) and the quality check was performed using FastQC. Reads from 552 FASTQ files were aligned to the $C$. trachomatis D serovar 434/Bu genome using alignment with 553 HISAT2. Differential gene regulation calculation was carried out based on feature Counts 554 algorithm. The differential gene expression heat map was created with Graph pad 7.

\section{Real Time PCR}

556 RNA was isolated from uninfected and Chlamydia-infected HeLa229 cells using RNA easy kit 557 (Qiagen, Germany). RNA was reverse transcribed using a Revert Aid First Strand synthesis Kit 
558 (Fermentas) according to the manufacturer's instructions and diluted 1:10 with RNase free

559 water. qPCR was performed as previously described ${ }^{42}$. Briefly, qPCR reactions were prepared 560 with Quanta SYBR (Quanta Bio) and PCR was performed on a Step One Plus device (Applied

561 Biosystems). Data was analysed using $\Delta$ Ct method, Step One Plus software package (Applied 562 Biosystems) and Excel (Microsoft). Endogenous control was GAPDH. Primers were designed

563 by q Primer Depot. The details of the primer are listed in the table in Extended data file.

\section{Transcervical mouse infections and determination of bacterial burden}

565 ASCT2/SLC1A5 KO mice generated in a C57BL/6 background were obtained from the 566 Australian National University (ANU) ${ }^{28}$. All animal experiments were performed in accordance

567 with protocols approved by animal care and experimentation of German Animal Protection

568 Law approved under the Animal (Scientific Procedures) Act 1986 (project license 55.2-2532-

569 2-762). The mice used for experiment were between 10-14 weeks old. Five days before 570 transcervical infection, mice were treated subcutaneously with $2.5 \mathrm{mg}$ of DepoProvera 571 (medroxy-progesterone acetate). The mice were transcervically infected with either $5721 \times 10^{7}$ infection-forming units (IFU) of $C$. trachomatis or $1 \times 10^{4} \mathrm{IFU}$ of $C$. muridarum using a 573 non-surgical embryo transfer device (ParaTechs Corp.). The mice were euthanized 7 days 574 post-infection and the uterine horns were taken for further analysis. The uterine horns were 575 homogenized in SPG buffer and DNA was isolated using DNeasy blood and tissue kit 576 (Qiagen). Quantitative PCR was used to enumerate Chlamydia and host genome copy 577 number. The following primers were used for amplifying the $C$. trachomatis lyt $A$ gene that 578 was cloned into the vector: fwd, 5'-TCTAAAGCGTCTGGTGAAAGCT-3' and rev, 5'579 GAAATAGCGTAGTAATAATACCCG-3'. Normalization of bacterial genome to that of the host 580 was performed using mouse synectin primers: fwd, 5'-ACTAATGTCAAGGAGCTGTACG-3' 581 and rev, 5'CCTCCGACTTGAACACTTCC-3'. Quantitative PCR with reverse transcription 582 (RT-PCR) was performed as described below. Data were analysed using Step One Plus 583 software package (Applied Biosystems) and expressed as the ratio of chlamydial genome to 584 host genome (lytA/synectin). GraphPad Prism 7 was used to generate a scatter column chart 
and perform statistical analysis. One-way analysis of variance (ANOVA) with Newman-Keuls

586 multiple-comparison tests was performed with the significance level set to less than 0.01 .

587 Statistical analysis was performed to decide the sample size used in mouse infection by the

588 Institute of Mathematics, University of Würzburg under the allowance A2 55.5-2531.01-49/12.

589 All mouse experiments were carried out with 9 or 10 female mice per treatment group. Mice

590 in each experiment were age-matched and cage mates were randomly distributed into

591 different treatment groups to avoid cage effects.

\section{Generation of mouse organoids}

593 Generation of mouse organoids was adapted from ${ }^{44}$. Mice were sacrificed and fallopian tube 594 tissue was prepared and processed within $2 \mathrm{~h}$. Briefly, tissue samples were washed with DPBS 595 (Gibco) and placed into a sterile Petri dish (Corning) where they were cut into small pieces. 596 Then, on the top of the minced tissue, a glass slide (VWR) was placed and strongly pressed 597 down to obtain smaller pieces. The cells were washed with DPBS, placed into a $15 \mathrm{ml}$ falcon 598 tube and centrifuged at $1,000 \mathrm{~g}$ for 10 minutes. The supernatant was removed, the pellet was 599 resuspended in Matrigel (Corning) and plated in $50 \mu \mathrm{l}$ drop in wells in a 24-well plate. The plate 600 was carefully transferred to $37^{\circ} \mathrm{C}$ incubator to allow the Matrigel to get solidified for $20 \mathrm{~min}$ 601 following the addition $500 \mu \mathrm{l} /$ well of pre-warmed media (DMEM advanced (Sigma), Wnt (25\%), 602 R-Spondin (25\%), Noggin (10\%), B27 (2\%; Thermo Scientific), Nicotinamide (1 mM; Sigma), 603 human EGF (50 ng/ml; Thermo Scientific), FGF (100 ng/ml; Thermo Scientific), TGF-ß inhibitor 604 (0.5 mM; Tocris), Rock inhibitor (10 mM; Abmole Bioscience).

605 Splitting organoids: Approximately in 7 days later, the drop was carefully resuspended in cold 606 DMEM medium and centrifuged at $1000 \mathrm{~g}$ at $4^{\circ} \mathrm{C}$ for $5 \mathrm{~min}$. The supernatant was discarded and $60750 \mu$ l Matrigel was added and further processed as explained above.

608 Infectivity assay in organoids: For infection with $C$. trachomatis mature organoids were 609 released from a confluent Matrigel drop by resuspending it with ice-cold DPBS (Gibco). The 610 suspension was collected in a low-binding Eppendorf tube and $2 \mu$ of a frozen stock of $C$. 611 trachomatis L2 expressing GFP was added ( $\left.5 \times 10^{5} \mathrm{IFU}\right)$. The suspension was mixed and placed 612 on ice for 30 min following centrifugation. $50 \mu \mathrm{l}$ of Matrigel was added to each tube and seeded 
613 into a 24 -well plate (Corning) with following 20 -minute incubation at $37^{\circ} \mathrm{C}$ to allow the Matrigel

614 drop solidify. 6 days post infection the organoids were fixed with 4\% PFA and used for

615 immunostaining. In addition, infected organoids were lysed with glass beads and different

616 dilutions were used to infect freshly plated HeLa cells to analyse the infectivity of the progeny.

617 Statistical analysis

618 In all experiments, a minimum of three technical replicates was used and the $n$ number refers

619 to the number of independent experiments performed. The data are presented as box plots 620 with the mean and s.e.m. Statistical analyses were performed with the Prism 7.2 package

621 (GraphPad Software). ExactTest () function as a part of edgeR module in R 3.3.4 was used to 622 carry out pairwise comparison and calculate $p$-values. False discovery rates were calculated as 623 q-values using Benjamini-Höchberg algorithm implemented in edgeR module.

\section{Author contribution}

$626 \mathrm{KR}$ and TR designed the experiments. The experiments were performed by KR, NV and TW.

627 Next generation sequencing on Illumina platform was performed by AB, EW and resulting RNA628 Seq data was analysed by RS and KR. Samples for Mass Spectrometry were prepared by KR 629 and SJ. NV, CH, MS and WE performed the metabolic flux analysis of axenic culture and data 630 interpretation. SJ, WS and AS performed metabolic flux analysis in host cell culture. FD provided 631 plasmids and cell lines. Click reagents were synthesized by JF and JS. KR and TR wrote the 632 manuscript.

\section{Acknowledgment}

634 We kindly acknowledge Stefan Broer and Anselm Enders, Australian National University for 635 providing the SLC1A5 KO mice. We thank Naziia Kurmasheva for supporting the organoid 636 experiment. We acknowledge Claudia Gehring and Daniela Bunsen for processing the TEM 637 samples. We thank Isabelle Derre for the EB-RB reporter strain $\mathrm{Ct}$ mCh(GroL2) GFP(OmcAL2), 638 Vera Kozjak-Pavlovic for the cell line expressing shRNA against GLS1 and Andreas Demuth 639 and Werner Goebel for critically reading the manuscript. KR was partially funded by the 640 Frauenbüro in the frame of the "Qualification for junior scientist for professorship programme" 
641 and by the Department of Biomedicine, Aarhus University. This research work was supported

642 by the German Research Foundation (DFG) grant WO 2108/1-1 to WE and the GRK 2157 "3D-

643 Infect" and the European Research Council (ERC-2018-ADG/NCI-CAD) to TR. AB was

644 supported by grants of the German Excellence Initiative to the Graduate School of Life Sciences,

645 University of Würzburg. SJ was supported by the DFG grant SCHU2670/1-1.

646 Data availability

647 Microarray data have been deposited in the Gene Expression Omnibus (GEO;

648 www.ncbi.nlm.nih.gov/geo/) of the National Center for Biotechnology Information and can be

649 accessed with the GEO accession numbers GSE147538 and GSE147539. Any other data that

650 support the findings of this study are available from the corresponding author upon request.

651 Code availability

652 Code described here has been integrated into the Galaxy codebase and released under the

653 Academic Free License (AFL) v. 3.0 (https://github.com/galaxyproject/galaxy

$654<$ https://github.com/galaxyproject/galaxy>). A free public instance of Galaxy can be accessed at 655 https://usegalaxy.org <https://usegalaxy.org/>. For the transcriptomics analysis on sequencing 656 data deposited at gene expression omnibus (GSE147538), open access code implemented in 657 bowtie, samtools, edgeR and GSEA tool and packages was utilized.

658 Declaration of interests

659 There is no conflict of interest

660

661 


\section{Figure legends}

663 Fig 1: Cell wall synthesis of Chlamydia grown in axenic medium is induced by glutamine.

664 a. Central metabolic pathways of the chlamydial and host TCA cycle. b. Diagram showing the setup of the experiment. c. The relative amounts of the indicated metabolites compared to time point 0 were determined in the supernatant of axenic cultures with Chlamydia at different time points by GC/MS using internal standards (see Methods). The stability of Gln is shown as the

668 green line where the supernatant with only Gln was analysed at 0 and $24 \mathrm{~h}$. Number of 669 independent experiments to confirm uptake: GIn: $n=6, G l u: n=5, K G: n=4$. d. ${ }^{13}$ C-enrichments 670 and isotopologue distributions of selected metabolites in Chlamydia cultured in axenic medium 671 with $\left[\mathrm{U}^{13} \mathrm{C}_{5}\right]$ Gln for 24 h. $\mathrm{n}=3$. e. Purified EBs (Input) were incubated in axenic medium with 672 only G6P or G6P together with GIn or Glu. After $24 \mathrm{~h}$, the bacteria were pelleted and analysed 673 by EM. Representative images from three independent experiments are shown. Scale bar $=2$ $674 \mu \mathrm{m}$. f. Purified EBs were incubated with G6P or G6P and Gln. Both samples were treated with 675 DA-DA for $12 \mathrm{~h}$. Click chemistry was used to detect DA-DA in peptidoglycan (green). Bacteria 676 were stained for cHsp60 (red). The samples were analysed using Structured Illumination 677 Microscopy. Representative images from two independent experiments. The insets show 678 magnifications of the peptidoglycan signal (lower panel). Scale bar $=0.5 \mu \mathrm{m}$. g. FACS analysis 679 of the bacteria from $(f)$. The fluorescence is presented as bar plot overlaid with data points. Data 680 were averaged ( \pm s.e.m.) from three independent experiments $(n=3)$. Statistical significance was 681 calculated using an unpaired t test (two tailed) $(t=5.138, D f=4, p$ value $=0.0068) .{ }^{* *}$ Indicates $p$ 682 value $<0.01$. h. EBs were incubated in axenic medium containing only G6P or G6P together with 683 Gln. After $12 \mathrm{~h}$ the bacteria were pelleted and total RNA was isolated for RNA-seq (see 684 Methods). The heat map shows expression levels of known early genes. i. Genome copy number of Chlamydia was determined at the indicated times. Shown is the mean from three independent experiments ( \pm s.e.m.) $n=3$. Statistical significance was calculated using two-way 687 ANOVA with Dunnett's multiple comparison test $(\mathrm{Df}=24) .{ }^{*} \mathrm{p}$ value $=0.034 ;{ }^{* *} \mathrm{p}$ value $=0.002$; ns, non-significant. 
690 Fig. 2: Chlamydial infection triggers altered glutamine metabolism in infected cells.

691 HUVECs were either left uninfected or infected with Chlamydia at an MOI of 1 for $36 \mathrm{~h}$. Cells or 692 medium samples were extracted and metabolites were analysed by LC-MS (see Methods). $\mathbf{a}$. 693 Depletion of amino acids from the culture medium from HUVECs with and without infection (only 694 significant results are shown). Data were averaged ( \pm s.e.m.) from three independent 695 experiments. $n=3$. b/c. Content of $G / n$ and Glu in control and infected cells. Data from three 696 independent experiments are depicted as bar plot overlaid with data points with the mean $( \pm$ 697 s.e.m.). Paired two tailed t-test was used to calculate statistical significance $(\mathbf{b}) t=10.75$, $D f=2$, $698 \mathrm{p}$ value $=0.0085 ;(\mathbf{c}) \mathrm{t}=1,664, \mathrm{Df}=2) \mathrm{p}$ value $=0.2379 .{ }^{* *} \mathrm{p}$ value $<0.01$, ns non-significant. Error 699 bar was defined as mean with SEM d. Control or Chlamydia infected HUVECs were cultured in 700 the presence of $\left[\mathrm{U}-{ }^{13} \mathrm{C}_{5}\right] \mathrm{G}$ In for $36 \mathrm{~h} .{ }^{13} \mathrm{C}$-enrichments and isotopologuedistributions of selected 701 metabolites were determined by LC-MS. Data present the mean of three independent samples.

702 e. Diagram outlining the synthesis of the D-aminopimelic acid (DAP) in Chlamydia from host or 703 chlamydial derived metabolites. f. Mass isotopologue distribution of DAP extracted from inf ected 704 host cells using acid hydrolysis. Data present the mean of three independent samples.

Fig. 3: Signalling pathways involved in the stabilization of c-Myc in infected cells. a. Plot of gene set enrichment analysis (GSEA) performed with RNA-seq data of the gene set „Positive regulation of c-myc targets“. Gene expression profiles were generated as described in the Methods section and a selected gene set is shown. Vertical black bars indicate the position of

710 genes in the „Positive regulation of c-myc targets“ gene set and the enrichment score is shown 711 as a green line. This analysis demonstrates that genes induced upon infection are known as 712 target genes of c-Myc (NES: normalized enrichment score). Three independent experiments 713 were performed. $n=3$. See the methods section for statistical analysis. b. Hela229 cells were 714 infected with Chlamydia for different time points and the samples were analysed using Western 715 blotting for phosphorylated c-Myc (pc-Myc serine 62), pc-Myc (threonine 58), cHsp60 and B 716 Actin. c. c-Myc was immunoprecipitated from lysates of HeLa229 cells inf ected with Chlamydia 717 for different time points. Ubiquitin was detected in the precipitate by immunoblotting. The input 
718 from the same experiment was probed against c-Myc, cHsp60 and B Actin. d. HeLa229 cells

719 were either left uninfected or infected with Chlamydia for 24 or 36 hpi. The cells were treated 720 with the translation inhibitor cycloheximide (CHX) for 10, 30 or $60 \mathrm{~min}$. The cells were then lysed 721 and analysed by Western blotting for c-Myc, cHsp60 and B Actin. For quantification, values were 722 normalized for each condition to the 0 time point of $\mathrm{CHX}$-treated sample. e. Diagram showing 723 the signalling pathway leading to c-Myc stabilization. $\mathbf{f} / \mathbf{g}$. HeLa229 cells were treated with the 724 MAPK inhibitor, (U0126) (f) or the PI3K inhibitor (LY294002) (g) for $4 \mathrm{~h}$ and infected with 725 Chlamydia for $24 \mathrm{~h}$. The cells were lysed and analysed by Western blotting. $\mathrm{n}=3$. h. HeLa229 726 cells were infected with Chlamydiafor different time points. The cells were harvested for Western 727 Blot analysis. $n=3$. i. HeLa229 cells were either left uninfected or infected with Chlamydia and 728 treated with the PI3K inhibitor. The cells were lysed and analysed by Western. In all Western 729 blots, detection of cHsp60 served as an infection and of B Actin as a loading control. The immunoblots shown in this figure are representative of at least three independent experiments.

732 Fig. 4: c-Myc is downstream of PI3K/MAPK pathway and critical for chlamydial growth.

$733 \mathbf{a} / \mathbf{b}$. U2OS cells harboring an inducible c-Myc gene under the control of a Tet-inducible promoter 734 were induced with AHT for $12 \mathrm{~h}$ and either treated with MAPK inhibitor U0126 (a), or the PI3K 735 inhibitor LY294002. n=3. (b) or left untreated as indicated. The cells were further infected with 736 Chlamydia and analysed by Western blotting for bacterial infection. $n=3$. c. The cells from the experiment shown in (a) and (b) were fixed and immunostained to detect chlamydial inclusions

738 (green) and actin (phalloidin, red). Images are representative for three independent 739 experiments. UI, uninfected control; U0: treated with U0126; LY, treated with LY294002; AHT, 740 induced. $\mathrm{n}=3$. Scale bar $=15 \mu \mathrm{m}$. d. HeLa229 cells were treated with the chemical c-Myc 741 inhibitor 10058-F4. The cells were infected with Chlamydia for 24 or 36 hpi and then analysed 742 for Chlamydia infection by Western blotting (cHsp60). $n=3$. e. HCT116 cells engineered to 743 express a shRNA to silence c-Myc expression under the control of an inducible Tet ${ }^{\text {on }}$ promoter 744 were treated with AHT $(1 \mu \mathrm{g} / \mathrm{ml})$ for $24 \mathrm{~h}$ and then infected with Chlamydia for another $24 \mathrm{~h}$. The

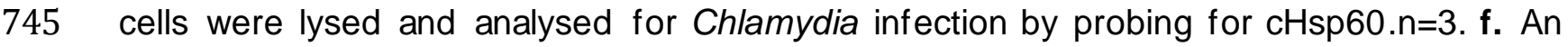


746 unrelated control siRNA or a siRNA pool directed against c-Myc was used to knock down c-Myc

747 in HeLa cells. After $48 \mathrm{~h}$ of transfection, the cells were infected with Chlamydia for $24 \mathrm{~h}$. The

748 cells were harvested and analysed by Western blotting for chlamydial infection. The 749 immunoblots shown in the figures are representative of at least three independent experiments.

751 Fig. 5: Glutamine is a limiting metabolite for chlamydial intracellular growth. a. Cells 752 isolated from human fimbriae (Human Fimb) were grown in basic formulation of DMEM 753 containing $1 \mathrm{~g} / \mathrm{l} \mathrm{D}$-glucose and different concentrations of glutamine. The cells were infected 754 with Chlamydia (Ct) for $24 \mathrm{~h}$, lysed and analysed via Western blotting to detect chlamydial 755 Hsp60, c-Myc and B Actin. $n=3$. b. HeLa229 cells were grown in basic formulation of DMEM containing $1 \mathrm{~g} / \mathrm{l} \mathrm{D}$-glucose. The cells were treated with or without glutamine $(2 \mathrm{mM})$ and infected with Chlamydiafor different time points. The cells were lysed and analysed for chlamydial growth using Western blotting to detect chlamydial Hsp60, c-Myc and B Actin. $n=3$. c/d. The cells from (b) were lysed and used to infect freshly plated HeLa cells. After $24 \mathrm{~h}$ the cells were either fixed and immunostained for chlamydial inclusions (green) and Actin (phalloidin, red), or used for Western blot analysis as shown in panel (d). The scale bar is indicated in the figure. $n=3$. Scale bar $=10 \mu \mathrm{m}$. e. HeLa cells were infected with the EB/RB reporter strain for $24 \mathrm{~h}$ and grown in basic formulation of DMEM containing $1 \mathrm{~g} / \mathrm{l}$ D-glucose either without (-GIn) or with $2 \mathrm{mM}$ Gln (+Gln). In one set of experiments, Gln-free medium was replaced after $24 \mathrm{~h}$ for medium containing $2 \mathrm{mM}$ Gln (lowest panel). The cells were fixed Chlamydia (Red/Green) and Actin (phalloidin, grey) was detected. Loss of the green fluorescence is indicative for RBs. $n=3$. Scale bar $=10 \mu \mathrm{m} . \mathbf{f} / \mathbf{g}$. HCT116 were grown in basic formulation of DMEM containing $1 \mathrm{~g} / \mathrm{l} \mathrm{D}$-glucose, with or without L-glutamine. HCT116 expressing 4-hydroxytamoxifen (OHT)-inducible Myc-ER or the vector control were treated overnight with OHT (100 nM) (f) to activate Myc-ER or ethanol

$770(1 \mu \mathrm{l})$ as control $(\mathbf{g})$. The cells were then infected with Chlamydia at an MOI of 1 for $24 \mathrm{~h}$ and 771 analysed for bacterial load by Western blotting. $n=3$. The immunoblots shown in the figures are 772 representative of at least three independent experiments. 
774 Fig. 6: Chlamydia metabolically reprograms the host cell. a. HUVEC cells infected with

775 Chlamydia were subjected to RNA-seq. Shown is the hierarchically clustered heat map depicting 776 gene regulation (normalized log2FC) of gene set "Amino acid transport across the plasma 777 membrane" which is a part of Reactome gene sets in C2 Molecular Signature Database. Three 778 independent experiments were performed. $n=3$. b. Diagram of Gln uptake pathways and the 779 targets of the inhibitors. c. HeLa229 cells were infected with Chlamydia and then subjected to 780 Western blot analysis. d. Control siRNA or an siRNA pool against SLC1A5 were transfected and $78148 \mathrm{~h}$ later infected with Chlamydia for $24 \mathrm{~h}$ as indicated. The cells were lysed and further 782 analysed by Western blot. e. HeLa229 cells expressing a shRNA to silence GLS1 expression 783 under the control of a Tet ${ }^{\text {on }}$ promoter were induced with AHT for 7 days to deplete GLS1 and 784 then subjected to Western blot analysis. f. HeLa229 cells were treated with the SLC1A5 specific 785 inhibitor GPNA or ( $\mathbf{g})$ the glutaminase inhibitor BPTES for $4 \mathrm{~h}$ and infected with Chlamydia. The 786 cells were lysed and analysed by Western blotting. h. HeLa229 cells were transfected with 787 siRNA against c-Myc and 48 hrs later analysed by Western blotting as indicated. i. HCT116 788 Tet $^{\text {on }}$ sh-c-Myc cells were either left untreated or treated with AHT for $24 \mathrm{~h}$ followed by 789 overexpression of SLC1A5. The cells were then infected with Chlamydia for another $24 \mathrm{~h}$ and 790 analysed by Western blotting. j/k. SLC1A5 +/+ or SLC1A5 -/- mice were infected with $C$. 791 trachomatis $(n=10)$ or $C$. muridarum $(n=9)(\mathbf{k})$. The mice were sacrificed seven days post 792 infection and the copy number of Chlamydia was calculated using qPCR. The data are 793 presented as box and whisker plot overlaid with data points with the mean ( \pm s.e.m.). Unpaired $794 \mathrm{t}$ test (two tailed) was used to calculate the significance $(\mathrm{j})(\mathrm{t}=3.714, \mathrm{df}=16)$ and $(\mathrm{k})(\mathrm{t}=3.712$, $795 \mathrm{df}=10) .{ }^{* *}$ Indicates $\mathrm{p}$ value $<0.01 . \mathrm{n}=9$. I. Organoids were derived from SLC1A5 +/+ or SLC1A5 796 -/- mice and infected with Chlamydia (Ct) (see Methods). The infected organoids were fixed and 797 used for immunostaining to detect Chlamydia (green), nuclei (blue) and actin (phalloidin, violet). 798 Scale bar $=30 \mu \mathrm{m}$. The immunoblots shown in the figures are representative of at least three 799 independent experiments. 
8021 Newman, L. et al. Global Estimates of the Prevalence and Incidence of Four Curable

803 Sexually Transmitted Infections in 2012 Based on Systematic Review and Global

804

805 Reporting. PLoS One 10, e0143304, doi:10.1371/journal.pone.0143304 (2015).

806

807 Liechti, G. W. et al. A new metabolic cell-wall labelling method reveals peptidoglycan in Chlamydia trachomatis. Nature 506, 507-510, doi:10.1038/nature12892 (2014).

3 Liechti, G. et al. Pathogenic Chlamydia Lack a Classical Sacculus but Synthesize a Narrow, Mid-cell Peptidoglycan Ring, Regulated by MreB, for Cell Division. PLoS Pathog 12, e1005590, doi:10.1371/journal.ppat.1005590 (2016).

4 Stephens, R. S. et al. Genome sequence of an obligate intracellular pathogen of humans: Chlamydia trachomatis. Science 282, 754-759 (1998).

5 Iliffe-Lee, E. R. \& McClarty, G. Glucose metabolism in Chlamydia trachomatis: the 'energy parasite' hypothesis revisited. Mol Microbiol 33, 177-187 (1999).

6 Mehlitz, A. et al. Metabolic adaptation of Chlamydia trachomatis to mammalian host cells. Mol Microbiol 103, 1004-1019, doi:10.1111/mmi.13603 (2017).

7 Omsland, A., Sager, J., Nair, V., Sturdevant, D. E. \& Hackstadt, T. Developmental stage-specific metabolic and transcriptional activity of Chlamydia trachomatis in an axenic medium. Proc Natl Acad Sci U S A 109, 19781-19785, doi:10.1073/pnas.1212831109 (2012).

8 Kubo, A. \& Stephens, R. S. Substrate-specific diffusion of select dicarboxylates through Chlamydia trachomatis PorB. Microbiology 147,3135-3140, doi:10.1099/00221287-147-11-3135 (2001).

9 Belland, R. J. et al. Genomic transcriptional profiling of the developmental cycle of Chlamydia trachomatis. Proc Natl Acad Sci U S A 100, 8478-8483, doi:10.1073/pnas.1331135100 (2003).

10 Belland, R. J. et al. Transcriptome analysis of chlamydial growth during IFNgamma-mediated persistence and reactivation. Proc Natl AcadSci U S A 100, 15971-15976, doi:10.1073/pnas.2535394100 (2003).

11 Miller, D. M., Thomas, S. D., Islam, A., Muench, D. \& Sedoris, K. c-Myc and cancer metabolism. Clin Cancer Res 18, 5546-5553, doi:10.1158/1078-0432.CCR-12-0977 (2012).

12 Gao, P.etal. c-Myc suppression of miR-23a/b enhances mitochondrial glutaminase expression and glutamine metabolism. Nature 458, 762-765, doi:10.1038/nature07823 (2009).

13 Wise, D. R. et al. Myc regulates a transcriptional program that stimulates mitochondrial glutaminolysis and leads to glutamine addiction. Proc Natl Acad Sci U S A 105, 18782-18787, doi:10.1073/pnas.0810199105 (2008).

14 Al-Zeer, M. A. et al. Chlamydia trachomatis Prevents Apoptosis Via Activation of PDPK1-MYC and Enhanced Mitochondrial Binding of Hexokinase II. EBioMedicine 23,100-110, doi:10.1016/j.ebiom.2017.08.005 (2017).

15 Welcker, M. et al. The Fbw7 tumor suppressor regulates glycogen synthase kinase 3 phosphorylation-dependent c-Myc protein degradation. Proc Natl Acad Sci U S A 101, 9085-9090, doi:10.1073/pnas.0402770101 (2004).

16 Sears, R. C. The life cycle of C-myc: from synthesis to degradation. Cell Cycle 3, 1133-1137 (2004). 
84717 Rajalingam, K. et al. Mcl-1 is a key regulator of apoptosis resistance in Chlamydia trachomatis-infected cells. PLoS One 3, e3102, doi:10.1371/journal.pone.0003102 (2008).

18 Subbarayal, P. et al. EphrinA2 receptor (EphA2) is an invasion and intracellular signaling receptor for Chlamydia trachomatis. PLoS Pathog 11, e1004846, doi:10.1371/journal.ppat.1004846 (2015).

19 Patel, A. L. et al. Activation of epidermal growth factor receptor is required for Chlamydia trachomatis development. BMC Microbiol 14, 277, doi:10.1186/s12866014-0277-4 (2014).

20 Adhikary, S. \& Eilers, M. Transcriptional regulation and transformation by Myc proteins. Nat Rev Mol Cell Biol 6, 635-645, doi:10.1038/nrm1703 (2005).

21 Frame, S., Cohen, P. \& Biondi, R. M. A common phosphate binding site explains the unique substrate specificity of GSK3 and its inactivation by phosphorylation. Mol Cell 7, 1321-1327 (2001).

22 Lorenzin, F. et al. Different promoter affinities account for specificity in MYCdependent gene regulation. Elife 5, doi:10.7554/eLife.15161 (2016).

23 Cortina, M. E., Ende, R. J., Bishop, R. C., Bayne, C. \& Derre, I. Chlamydia trachomatis and Chlamydia muridarum spectinomycin resistant vectors and a transcriptional fluorescent reporter to monitor conversion from replicative to infectious bacteria. PLoS One 14, e0217753, doi:10.1371/journal.pone.0217753 (2019).

24 Dejure, F. R. et al. The MYC mRNA 3'-UTR couples RNA polymerase II function to glutamine and ribonucleotide levels. The EMBO journal 36, 1854-1868, doi:10.15252/embj.201796662 (2017).

25 Kekuda, R. et al. Cloning of the sodium-dependent, broad-scope, neutral amino acid transporter Bo from a human placental choriocarcinoma cell line. J Biol Chem 271, 18657-18661 (1996).

26 van Geldermalsen, M. et al. ASCT2/SLC1A5 controls glutamine uptake and tumour growth in triple-negative basal-like breast cancer. Oncogene 35, 3201-3208, doi:10.1038/onc.2015.381 (2016).

27 Wise, D. R. et al. Myc regulates a transcriptional program that stimulates mitochondrial glutaminolysis and leads to glutamine addiction. P Natl Acad Sci USA 105, 18782-18787, doi:10.1073/pnas.0810199105 (2008).

28 Masle-Farquhar, E., Broer, A., Yabas, M., Enders, A. \& Broer, S. ASCT2 (SLC1A5) Deficient Mice Have Normal B-Cell Development, Proliferation, and Antibody Production. Front Immunol 8, 549, doi:10.3389/fimmu.2017.00549 (2017).

29 Kermack, A. J. et al. Amino acid composition of human uterine fluid: association with age, lifestyle and gynaecological pathology. Hum Reprod 30, 917-924, doi:10.1093/humrep/dev008 (2015).

30 Behjousiar, A., Kontoravdi, C. \& Polizzi, K. M. In situ monitoring of intracellular glucose and glutamine in CHO cell culture. PLoS One 7, e34512, doi:10.1371/journal.pone.0034512 (2012).

31 Allan, I. \& Pearce, J. H. Differential amino acid utilization by Chlamydia psittaci (strain guinea pig inclusion conjunctivitis) and its regulatory effect on chlamydial growth. J Gen Microbiol 129, 1991-2000, doi:10.1099/00221287-129-7-1991 (1983).

32 Allan, I. \& Pearce, J. H. Amino acid requirements of strains of Chlamydia trachomatis and C. psittaci growing in McCoy cells: relationship with clinical syndrome and host origin. J Gen Microbiol 129, 2001-2007, doi:10.1099/00221287-129-7-2001 (1983). 
33 Jin, L., Alesi, G. N. \& Kang, S. Glutaminolysis as a target for cancer therapy. Oncogene 35, 3619-3625, doi:10.1038/onc.2015.447 (2016).

34 Porcheray, F. et al. Glutamate metabolism in HIV-infected macrophages: implications for the CNS. Am J Physiol Cell Physiol 291, C618-626, doi:10.1152/ajpcell.00021.2006 (2006).

35 Chambers, J. W., Maguire, T. G. \& Alwine, J. C. Glutamine metabolism is essential for human cytomegalovirus infection. J Virol 84, 1867-1873, doi:10.1128/JVI.0212309 (2010).

36 Fontaine, K. A., Camarda, R. \& Lagunoff, M. Vaccinia virus requires glutamine but not glucose for efficient replication. J Virol 88, 4366-4374, doi:10.1128/JVI.0313413 (2014).

37 Thai, M. et al. Adenovirus E4ORF1-induced MYC activation promotes host cell anabolic glucose metabolism and virus replication. Cell Metab 19, 694-701, doi:10.1016/j.cmet.2014.03.009 (2014).

38 Thai, M. et al. MYC-induced reprogramming of glutamine catabolism supports optimal virus replication. Nat Commun 6, 8873, doi:10.1038/ncomms9873 (2015).

39 Sanchez, E. L., Carroll, P. A., Thalhofer, A. B. \& Lagunoff, M. Latent KSHV Infected Endothelial Cells Are Glutamine Addicted and Require Glutaminolysis for Survival. PLoS Pathog 11, e1005052, doi:10.1371/journal.ppat.1005052 (2015).

40 Mall, A. et al. Reversibility of citrate synthase allows autotrophic growth of a thermophilic bacterium. Science 359, 563-567, doi:10.1126/science.aao2410 (2018).

41 Lee, W. N., Byerley, L. O., Bergner, E. A. \& Edmond, J. Mass isotopomer analysis: theoretical and practical considerations. Biol Mass Spectrom 20, 451-458, doi:10.1002/bms.1200200804 (1991).

42 Karunakaran, K., Subbarayal, P., Vollmuth, N. \& Rudel, T. Chlamydia-infected cells shed Gp96 to prevent chlamydial re-infection. Mol Microbiol 98, 694-711, doi:10.1111/mmi.13151 (2015).

43 Subramanian, A. et al. Gene set enrichment analysis: a knowledge-based approach for interpreting genome-wide expression profiles. Proc Natl AcadSci U S A 102, 15545-15550, doi:10.1073/pnas.0506580102 (2005).

44 Kessler, M. et al. Chronic Chlamydia infection in human organoids increases stemness and promotes age-dependent CpG methylation. Nat Commun 10, 1194, doi:10.1038/s41467-019-09144-7 (2019). 